\title{
Macroscopic Findings Specimen Type
}

National Cancer Institute

\section{Source}

National Cancer Institute. Macroscopic Findings Specimen Type. NCI Thesaurus. Code C119871.

The type of material sample taken from a biological entity for macroscopic findings assessment testing. 